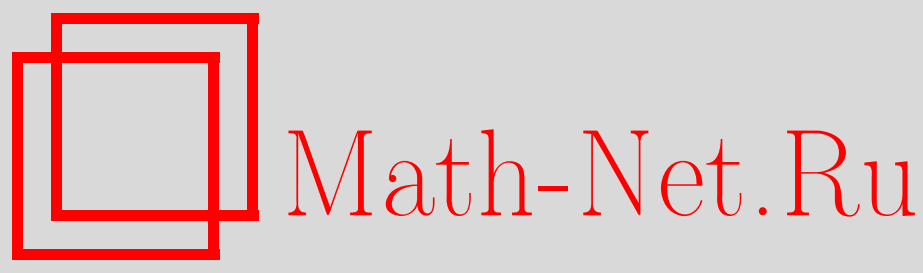

Ю. Г. Рудой, Метрика Боголюбова как глобальная характеристика семейства метрик в гильбертовой алгебре наблюдаемых, ТМФ, 2009, том 160, номер 2, 352-369

DOI: https://doi.org/10.4213/tmf6403

Использование Общероссийского математического портала Math-Net.Ru подразумевает, что вы прочитали и согласны с пользовательским соглашением http://www . mathnet.ru/rus/agreement

Параметры загрузки:

IP: 35.174 .16 .151

26 апреля 2023 г., 15:28:16

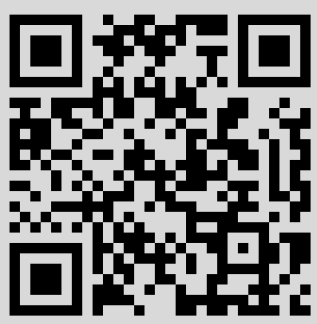




\title{
ФИЗИКА
}

Том 160, № 2

август, 2009

2009 г.

Ю. Г. Рудой*

\section{МЕТРИКА БОГОЛЮБОВА КАК ГЛОБАЛЬНАЯ ХАРАКТЕРИСТИКА СЕМЕЙСТВА МЕТРИК В ГИЛЬБЕРТОВОЙ АЛГЕБРЕ НАБЛЮДАЕМЫХ}

\begin{abstract}
Проведен сравнительный анализ однопараметрического семейства билинейных комплексных функционалов, имеющих смысл "деформированных" скалярных произведений Вигнера-Яназе-Дайсона, на гильбертовой алгебре операторов физических наблюдаемых. Установлены зависимость этих функционалов и соответствующих им метрик от параметра деформации, а также экстремальные свойства метрик Кубо-Мартина-Швингера и Вигнера-Яназе в квантовой статистической механике. Показано, что метрика Боголюбова-Кубо-Мори является глобальной (интегральной) характеристикой указанного семейства. Она занимает промежуточное положение между экстремальными метриками и имеет ясный физический смысл обобщенной изотермической восприимчивости. Рассмотрен пример для алгебры наблюдаемых $S U(2)$ в простейшей модели идеального квантового спинового парамагнетика.
\end{abstract}

Ключевые слова: операторные метрики, корреляционные функции, функции Грина, спектральная интенсивность, соотношения неопределенностей.

\section{1. ВВЕДЕНИЕ. ОСНОВНЫЕ ОПРЕДЕЛЕНИЯ}

Важной задачей квантовой механики является вычисление корреляционных функций (вообще говоря, комплексных) как в чистых, так и в смешанных состояниях физических величин, которые описываются линейными операторами $A, B$. Эти операторы могут быть неограниченными (например, операторы координат или импульсов частиц), не коммутировать друг с другом и даже не являться самосопряженными $\left(A \neq A^{\dagger}, \ldots\right.$, знак $\dagger$ означает эрмитово сопряжение).

Операторы $A, B, \ldots$ действуют в гильбертовом пространстве состояний адиабатически изолированной физической системы, которая характеризуется эрмитовым оператором Гамильтона $H=H^{\dagger}$, причем совокупность операторов $A, B, H, \ldots$ образует алгебру наблюдаемых с инволюцией и единицей. Именно операторный, или алгебраический, подход считается наиболее удобным для формулировки квантовой статистической механики (см., например, [1]-[4]).

* Российский университет дружбы народов, Москва, Россия. E-mail: rudikar@mail.ru 
Для физической системы, находящейся в состоянии теплового равновесия с термостатом с фиксированной температурой Кельвина $0 \leqslant T<\infty$, справедливо квантовое обобщение подхода Гиббса, построенное фон Нейманом [5]. Состояние системы характеризуется тогда оператором плотности $e^{\Phi(\beta)-\beta H}$, где $\beta=1 / k_{\mathrm{B}} T \geqslant 0-$ обратная температура в энергетических единицах, $k_{\mathrm{B}}$ - постоянная Больцмана, $\Phi(\beta)=\ln Z(\beta)$ - характеристическая функция Масье-Планка, причем статистическая сумма $Z(\beta)=\operatorname{Sp} \rho(\beta)$. В дальнейшем для простоты используется ненормированный аналог положительно-определенного и ограниченного оператора плотности фон Неймана $\rho(\beta)=\rho^{\dagger}(\beta)=e^{-\beta H}$.

Среднее значение любого оператора $A$ (также ненормированное) имеет вид

$$
\langle A\rangle=\operatorname{Sp} A(\beta), \quad A(\beta)=\rho(\beta) A, \quad A(0)=A,
$$

где предполагается, что $A(\beta)$, как и $\rho(\beta)$, является ядерным оператором с конечным следом. Для этого необходимо, чтобы $A$ являлся оператором Гильберта-Шмидта, поскольку оператор $\rho(\beta)$ при $\beta>0$ ограничен, $0 \leqslant\|\rho(\beta)\| \leqslant 1$. Это имеет место, если $A$ ограничен; в противном случае можно использовать известный прием перехода к ограниченным функциям оператора $A$ (см. [1]-[4]).

По аналогии с (1) корреляционная функция $K_{A B}(\beta)$ для пары операторов $A$ и $B$ определяется билинейным комплексным функционалом

$$
K_{A B}(\beta) \equiv\left\langle A B^{\dagger}\right\rangle=\operatorname{Sp} A(\beta) B^{\dagger}=\operatorname{Sp} B^{\dagger} A(\beta)
$$

в частности, при $B=B^{\dagger}=I$, где $I$ - единичный оператор, функционал $K_{A I}(\beta)=$ $\langle A\rangle$, как и должно быть, переходит в (1). Предполагается, что $A$ и $B$ являются операторами Гильберта-Шмидта, так что произведение $A(\beta) B^{\dagger}$ является ядерным оператором и выражение (2) имеет смысл.

Это выражение задает в алгебре операторов $A, B, \ldots$ внутреннее, или скалярное, произведение (см., например, [6]), делающее эту алгебру нормированной гильбертовой алгеброй (вообще говоря, бесконечномерной); величина $K_{A A}(\beta)>0$ определяет в ней норму оператора $A$ и, соответственно, метрику $\left[K_{A A}(\beta)\right]^{1 / 2}$.

Стандартная метрика (2) типа Гильберта-Шмидта, введенная в [5], [7]-[9] на раннем этапе развития квантовой статистической механики, в физических приложениях впоследствии получила наименование метрики Кубо-Мартина-Швингера (КМШ) [10], [11]; эта метрика является наиболее простой и естественной, однако отнюдь не единственно возможной.

Интерес к применению обобщенных, или деформированных, метрик возник, в частности, в связи с теорией линейной реакции на термические возмущения, а также в связи с проблемой минимизации соотношений неопределенностей $(\mathrm{CH})$. В наиболее общей форме СН установлены Шредингером, где в дополнение к обычному $\mathrm{CH}$ в форме Гейзенберга учитывается вклад не только коммутатора операторов $A$ и $B$, но и вклад их антикоммутатора.

Примером $\lambda$-деформированных скалярных произведений является однопараметрическое семейство Вигнера-Яназе-Дайсона (ВЯД) $(0 \leqslant \lambda \leqslant 1)$, предложенное Дайсоном в работе [12] в качестве обобщения скалярного произведения Вигнера- 
Яназе (ВЯ) $(\lambda=1 / 2)$ :

$$
\begin{gathered}
K_{A B}^{\mathrm{WYD}}(\beta ; \lambda)=\left\langle A(\beta ; \lambda) B^{\dagger}\right\rangle=\operatorname{Sp} A(\beta ; \lambda) B^{\dagger}, \\
A(\beta ; \lambda)=[\rho(\beta)]^{1-\lambda} A[\rho(\beta)]^{\lambda}, \quad \rho(\beta)=\rho^{\dagger}(\beta)=e^{-\beta H}
\end{gathered}
$$

(в очевидных случаях индекс WYD будет опускаться).

На физическом уровне строгости предполагается следующее: а) $\lambda$-деформация операторов $A$ не выводит их из класса Гильберта-Шмидта; б) существуют дробные (неотрицательные) степени оператора $\rho(\beta)$; в) с учетом свойства $\rho(0)=I$ при $\beta=0$ существует конечное значение $K_{A B}(0 ; \lambda)=\operatorname{Sp} A B^{\dagger}$, не зависящее от $\lambda$. Последнее гарантирует существование $K_{A B}(\beta ; \lambda)$ при всех $\lambda$ и $\beta>0$, поскольку $\left|K_{A B}(\beta ; \lambda)\right| \leqslant$ $\left|K_{A B}(0 ; \lambda)\right|$ в силу ограниченности оператора $\rho(\beta)$.

При указанных предположениях аксиоматика скалярного произведения по-прежнему выполняется, причем выражение (3) допускает эквивалентную запись через коммутаторы операторов $A$ и $B^{\dagger}$ с дробными степенями $\rho(\beta)$ :

$$
K_{A B}(\beta ; \lambda)=K_{A B}(\beta ; 0)+\frac{1}{2} \operatorname{Sp}\left\{\left[(\rho(\beta))^{\lambda}, A\right]_{-}\left[(\rho(\beta))^{1-\lambda}, B^{\dagger}\right]_{-}\right\} .
$$

Подобная запись вводилась в работе [12] и далее использовалась рядом авторов, в том числе Либом [13], Бауманом и Иостом [14], для изучения свойства выпуклости $K_{A A}(\beta ; \lambda)$ по операторному аргументу $\rho(\beta)$.

Предельному значению $\lambda=0$ в (3) соответствует максимально несимметричная по $\lambda$ метрика КМШ $K_{A B}^{\mathrm{KMS}}(\beta ; 0)$, тогда как значению $\lambda=1 / 2$ - максимально симметричная метрика ВЯ $K_{A B}^{\mathrm{WY}}(\beta ; 1 / 2)$; промежуточные значения $0<\lambda<1 / 2$ были рассмотрены Латтинжером [15], причем все эти скалярные произведения являются локальными для семейства (3).

В 1961 году в работе [16] (гл. 1, § 5) Боголюбов ввел новое скалярное произведение (метрику) на алгебре наблюдаемых, которое, как оказалось, можно связать с семейством метрик (3). Именно, величина $K_{A B}^{\mathrm{BKM}}(\beta)$ для любой пары операторов $A$ и $B$ выражается интегралом $\int_{0}^{1} d \lambda K_{A B}(\beta ; \lambda)$; тем самым $K_{A B}^{\mathrm{BKM}}(\beta)$ играет роль глобальной характеристики для семейства $K_{A B}^{\mathrm{WYD}}(\beta ; \lambda)$ из $(3)$. Заметим, что логика введения Боголюбовым новой метрики базировалась на совершенно ином подходе, а именно на подходе функций Грина, который был развит им же (совместно с Тябликовым) несколько ранее [17] (подробнее см. ниже раздел 3).

Существенно, что метрика Боголюбова совпала с выражением, введенным несколько ранее независимо Кубо [10] и Мори [18]: оказалось, что величина $K_{A B}^{\mathrm{BKM}}(\beta)$ имеет ясный физический смысл и описывает обобщенную изотермическую восприимчивость $\chi_{A B}(\beta)$ (подробнее см. ниже конец раздела 2). Возможно, именно с этим фактом связано замечание Стритера [19] о том, что метрика Боголюбова-Кубо-Мори (БКМ) предпочтительна по сравнению с метрикой КМШ (а также, по-видимому, и со всеми остальными $\lambda$-метриками).

Цель данной работы состоит в том, чтобы дать сравнительное описание всего семейства $\lambda$-деформированных метрик, основанных на скалярном произведении (3), а также метрики БКМ, которая не принадлежит этому семейству, но порождается им. Основное внимание уделено обобщенному СН в форме Шредингера для пары 
операторов $A$ и $B$, которое выражается неравенством Коши-Буняковского-Шварца (КБШ), справедливому как для функционала (2), так и для его $\lambda$-деформированного аналога (3).

При вычислениях использовано полученное Латтинжером [15] обобщение на случай $\lambda \neq 0$ спектрального представления для $K_{A B}(\beta ; \lambda)$, впервые введенного Кэлленом и Вельтоном [20], а также метод функций Грина в формулировке Боголюбова и Тябликова [17] (см. также [21], [22]).

\section{2. ОБЩИЕ СВОЙСТВА $\lambda$-ДЕФОРМИРОВАННОГО СКАЛЯРНОГО ПРОИЗВЕДЕНИЯ}

Будем далее предполагать, что ни $A$, ни $B^{\dagger}$ не являются интегралами движения, причем коммутатор $\left[A, B^{\dagger}\right]_{-}$может быть как оператором, так и комплексным числом, в том числе равным нулю. Указанное ограничение обусловлено тем, что если хотя бы один из операторов $A$ или $B^{\dagger}$ коммутирует с гамильтонианом $H$, то $\lambda$-деформация (3) становится тривиальной и $K_{A B}(\beta ; \lambda)=K_{A B}(\beta ; 0)$. Действительно, если $[A, H]_{-}=0$, то это очевидно, поскольку $A(\beta ; \lambda)=A(\beta ; 0)$, а если $\left[B^{\dagger}, H\right]_{-}=0$, то следует учесть циклическую инвариантность следа.

Одно из важных общих свойств, присущих величине (3) как скалярному произведению, связано с эрмитовым сопряжением аргумента $A(\beta ; \lambda) B^{\dagger}$ :

$$
K_{B A}(\beta ; \lambda)=K_{A B}^{*}(\beta ; \lambda), \quad K_{A A}(\beta ; \lambda) \geqslant 0,
$$

где учтено, что оператор $[\rho(\beta)]^{\alpha}$ эрмитов при любом $\alpha \geqslant 0$ и что $\operatorname{Sp} X Y=\operatorname{Sp} Y X$ и $\operatorname{Sp} X^{\dagger}=(\operatorname{Sp} X)^{*}$ для операторов $X$ и $Y$ из класса Гильберта-Шмидта. Заметим, что с физической точки зрения $K_{A A}(\beta ; \lambda)$ представляет собой квантовую автокорреляционную функцию, всегда неотрицательную в силу (4).

Дополнительные свойства, связанные с зависимостью $K_{A B}(\beta ; \lambda)$ от вещественного параметра $\lambda$, можно получить, если учесть, что $[A(\beta ; \lambda)]^{\dagger}=A^{\dagger}(\beta ; 1-\lambda)$. Тогда имеем

$$
\begin{aligned}
& K_{A^{\dagger} B}(\beta ; \lambda)=K_{B^{\dagger} A}(\beta ; 1-\lambda)=K_{A B^{\dagger}}^{*}(\beta ; 1-\lambda), \\
& K_{A B^{\dagger}}(\beta ; \lambda)=K_{B A^{\dagger}}(\beta ; 1-\lambda)=K_{A^{\dagger} B}^{*}(\beta ; 1-\lambda),
\end{aligned}
$$

а также

$$
K_{A^{\dagger} B^{\dagger}}(\beta ; \lambda)=K_{B A}(\beta ; 1-\lambda)=K_{A B}^{*}(\beta ; 1-\lambda),
$$

где использована, например, цепочка равенств, приводящая к $(5): K_{B A}(\beta ; \lambda)=$ $\operatorname{Sp} B(\beta ; \lambda) A^{\dagger}=\operatorname{Sp}[A(\beta ; \lambda)]^{\dagger} B=\operatorname{Sp} A^{\dagger}(\beta ; 1-\lambda) B=K_{A^{\dagger} B}(\beta ; 1-\lambda)=K_{A B}^{*}(\beta ; \lambda)$.

Другим важным свойством величины (3) как скалярного произведения является неравенство КБШ [4], которое удобно записать, используя всегда неотрицательную КБШ-разностъ:

$$
\Delta_{A B}(\beta ; \lambda) \equiv K_{A A}(\beta ; \lambda) K_{B B}(\beta ; \lambda)-\left|K_{A B}(\beta ; \lambda)\right|^{2}, \quad \Delta_{A B}(\beta ; \lambda) \geqslant 0 .
$$

Физический интерес представляет вопрос о том, влияет ли значение $\lambda$ на величину (6) и, в частности, возможно ли при данных $A, B$ и $\lambda$ получить значение $\Delta_{A B}(\beta ; \lambda)=0$, т.е. насыщение неравенства КБШ. 
Ограничимся в дальнейшем классом эрмитовых операторов $A=A^{\dagger}, B=B^{\dagger}$, для которых из (5) следует важное свойство симметрии $K_{A B}(\beta ; \lambda)=K_{B A}(\beta ; 1-\lambda)$. В частности, при $A=B$ получаем для нормы оператора (метрики) $K_{A A}(\beta ; \lambda)=$ $K_{A A}(\beta ; 1-\lambda)$. Это свойство позволяет изучать $K_{A A}(\beta ; \lambda)$ только на отрезке $0 \leqslant \lambda \leqslant$ $1 / 2$, причем метрики КМШ и ВЯ являются тогда граничными для семейства (3), а метрика БКМ сохраняет для этого семейства смысл интегральной характеристики.

Произведение некоммутирующих эрмитовых операторов $A$ и $B$ не является эрмитовым, поэтому квантовая корреляционная функция $K_{A B}(\beta ; \lambda)$ при $A \neq B$ остается комплексной, так что $K_{A B}(\beta ; \lambda)=K_{A B}^{(+)}(\beta ; \lambda)+i K_{A B}^{(-)}(\beta ; \lambda)$; с учетом свойства симметрии (5) вещественная и мнимая части $K_{A B}^{( \pm)}(\beta ; \lambda)$ могут быть записаны в виде

$$
\begin{aligned}
& K_{A B}^{(+)}(\beta ; \lambda)=\frac{1}{2}\left\{K_{A B}(\beta ; \lambda)+K_{B A}(\beta ; \lambda)\right\} \\
& K_{A B}^{(-)}(\beta ; \lambda)=\frac{1}{2 i}\left\{K_{A B}(\beta ; \lambda)-K_{B A}(\beta ; \lambda)\right\} .
\end{aligned}
$$

С помощью (7) выражение (6) для КБШ-разности можно записать в виде

$$
\Delta_{A B}(\beta ; \lambda) \equiv K_{A A}(\beta ; \lambda) K_{B B}(\beta ; \lambda)-\left\{\left[K_{A B}^{(+)}(\beta ; \lambda)\right]^{2}+\left[K_{A B}^{(-)}(\beta ; \lambda)\right]^{2}\right\}
$$

более удобном для применений, поскольку одна из величин $K_{A B}^{(+)}(\beta ; \lambda)$ или $K_{A B}^{(-)}(\beta ; \lambda)$ часто оказывается равной нулю.

Величины $K_{A B}^{(\eta)}(\beta ; \lambda), \eta= \pm 1$, выражаются через коммутаторы $(\eta=-1)$ или антикоммутаторы $(\eta=+1) \lambda$-деформированных операторов $A(\beta ; \lambda) B+\eta B(\beta ; \lambda) A$; последние, однако, не выражаются в замкнутом виде через коммутаторы или антикоммутаторы исходных операторов $[A, B]_{\eta} \equiv A B+\eta B A$ (пусть даже и $\lambda$-деформированные). Подобное выражение оказывается возможным лишь в предельных случаях $\lambda=0$ или $\lambda=1$ (для них имеет место верхний или нижний знак соответственно):

$$
K_{A B}^{(+)}(\beta)= \pm \frac{1}{2}\left\langle[A, B]_{+}\right\rangle, \quad K_{A B}^{(-)}(\beta)= \pm \frac{1}{2 i}\left\langle[A, B]_{-}\right\rangle .
$$

Заметим, что коммутатор и антикоммутатор $[A, B]_{\eta}$ пары эрмитовых операторов являются соответственно эрмитовым и антиэрмитовым операторами, $\left([A, B]_{\eta}\right)^{\dagger}=$ $\eta[A, B]_{\eta}$

Рассмотрим далее общие свойства гладкости квантового коррелятора $K_{A B}(\beta ; \lambda)$ как непрерывной функции параметра $\lambda$. Дифференцируемость $K_{A B}(\beta ; \lambda)$ обусловлена наличием для дробных степеней ${ }^{1)}$ оператора $\rho(\beta)$ (непрерывных по $\lambda$ ) операторных уравнений Блоха:

$$
\frac{1}{\beta} \frac{d}{d \lambda} \rho^{\lambda}=\rho^{\lambda} H, \quad \frac{1}{\beta} \frac{d}{d \lambda} \rho^{1-\lambda}=-H \rho^{1-\lambda} .
$$

Очевидно, что операция дифференцирования по $\lambda$ сохраняет общую структуру (3).

\footnotetext{
1) Здесь учтено, что параметр $\lambda$ входит в экспоненциальный оператор $\rho^{\lambda}$ или $\rho^{1-\lambda}$ в мультипликативной форме.
} 
Обозначая указанную операцию штрихом и используя перестановочность операций $d / d \lambda$ и $\mathrm{Sp}$ (что предполагает равномерную сходимость ряда $(3))$, имеем $\left.^{2}\right)$

$$
\begin{gathered}
K_{A B}^{\prime}(\beta ; \lambda)=K_{C B}(\beta ; \lambda), \quad K_{A B}^{\prime \prime}(\beta ; \lambda)=K_{D B}(\beta ; \lambda), \quad \ldots, \\
C=[A, H]_{-}, \quad D=[C, H]_{-}=\left[[A, H]_{-}, H\right]_{-}, \quad \ldots,
\end{gathered}
$$

где коммутаторы $A$ с $H$ возрастающего порядка $C, D, \ldots$ представляют собой правые части динамических уравнений движения того же порядка для оператора $A$ и обращаются в нуль, если $A$ является интегралом движения и коммутирует с $H$.

Для выражения (3) существует и обратная к (11) операция интегрирования по $\lambda^{3)}$ :

$$
\int d \lambda K_{A B}(\beta ; \lambda)=K_{F B}(\beta ; \lambda), \quad A=[F, H]_{-},
$$

так что выражение для скалярного произведения (метрики) БКМ принимает вид

$$
K_{A B}^{\mathrm{BKM}}(\beta) \equiv \chi_{A B}(\beta)=\int_{0}^{1} d \lambda K_{A B}(\beta ; \lambda)=K_{F B}(\beta ; 1)-K_{F B}(\beta ; 0) .
$$

Величина $\chi_{A B}(\beta)$ согласно Кубо [10] и Мори [18] представляет собой изотермический "отклик" физической системы, состояние которой описывается оператором $\rho(\beta)=e^{-\beta H}$, на малое возмущение гамильтониана $H \rightarrow H(\varepsilon)=H+\varepsilon A$ $(\varepsilon \ll 1)$. В качестве "отклика" обычно рассматривается вариация среднего значения $\delta\left\langle B^{\dagger}\right\rangle=\operatorname{Sp} \delta \rho(\beta) B^{\dagger}$, где $\delta \rho(\beta)=e^{-\beta H(\varepsilon)}-e^{-\beta H}$. В низшем по $\varepsilon$ приближении имеем $\delta\left\langle B^{\dagger}\right\rangle \approx \chi_{A B}(\beta)$, что и является определением обобщенной изотермической $(\beta=$ const $)$ восприимчивости $\chi_{A B}(\beta)$. С другой стороны, разность экспоненциальных операторов $\delta \rho(\beta)$ в том же низшем приближении выражается интегралом Дюамеля (см., например, [6]), где $d H(\varepsilon) / d \varepsilon=A$, откуда для $\chi_{A B}(\beta)$ следует выражение (13).

\section{3. СПЕКТРАЛЬНОЕ ПРЕДСТАВЛЕНИЕ $\lambda$-ДЕФОРМИРОВАННОГО СКАЛЯРНОГО ПРОИЗВЕДЕНИЯ}

Удобным средством изучения общих выражений (3) и (11) для $\lambda$-деформированного скалярного произведения и его производных является спектральное представление величины $K_{A B}(\beta ; \lambda)$, впервые полученное в работе [15]:

$$
K_{A B}(\beta ; \lambda)=\int_{-\infty}^{\infty} d \omega J_{A B}(\omega ; \beta, \lambda), \quad J_{A B}(\omega ; \beta, \lambda)=J_{A B}(\omega ; \beta, 0) e^{\lambda \beta \hbar \omega},
$$

где $\omega$ - вещественная частота, $\hbar$ - постоянная Планка. Подобно $K_{A B}(\beta ; \lambda)$, спектральная интенсивность $J_{A B}(\omega ; \beta, \lambda)$ является комплексным функционалом.

Как показано Боголюбовым в работе [16] для случая $\lambda=0$, величина $J_{A B}(\omega ; \beta, 0)$ подобно $K_{A B}(\beta ; 0)$ обладает всеми свойствами скалярного произведения и играет ключевую роль в методе функций Грина, развитом Боголюбовым и Тябликовым [17] и в принципе позволяющим найти искомую величину $J_{A B}(\omega ; \beta, 0)$.

\footnotetext{
2) Более подробно вид и свойства этих производных будут изучены ниже в разделе 3.

3) Выражение (12) называют иногда тождеством Кубо или интегральным представлением коммутатора.
} 
Существенно, что $\lambda$-деформация оставляет в силе все выводы, полученные в работе [16], поскольку вся зависимость от $\lambda$ в (14) представлена только вещественным и положительным множителем $\left.{ }^{4}\right) e^{\lambda \beta \hbar \omega} \equiv e^{2 \lambda \theta}$, где $\theta=\beta \hbar \omega / 2$; очевидно, что $J_{A B}^{(n)}(\omega ; \beta, \lambda)=(2 \theta)^{n} J_{A B}(\omega ; \beta, \lambda)$, где $n-$ порядок производной по $\lambda$.

Используя представление (14) в выражениях для низших производных $K_{A B}(\beta ; \lambda)$, получаем в правых частях (9) вместо повторных коммутаторов низшие частотные моменты функции $J_{A B}(\omega ; \beta, \lambda)$ :

$$
\begin{aligned}
\frac{1}{\beta \hbar} K_{A B}^{\prime}(\beta ; \lambda) & =\int_{-\infty}^{\infty} d \omega \omega J_{A B}(\omega ; \beta, \lambda) \equiv \bar{\omega}(\beta, \lambda), \\
\left(\frac{1}{\beta \hbar}\right)^{2} K_{A B}^{\prime \prime}(\beta ; \lambda) & =\int_{-\infty}^{\infty} d \omega \omega^{2} J_{A B}(\omega ; \beta, \lambda) \equiv \overline{\omega^{2}}(\beta, \lambda) ;
\end{aligned}
$$

исходное выражение (14) имеет смысл $\overline{\omega^{0}}$.

Аналогично, интегрируя (14) по $\lambda$, получаем “обратный" момент:

$$
\beta \hbar \int d \lambda K_{A B}(\beta ; \lambda)=\int_{-\infty}^{\infty} d \omega \omega^{-1} J_{A B}(\omega ; \beta, \lambda) \equiv \overline{\omega^{-1}}(\beta, \lambda),
$$

откуда находим

$$
K_{A B}^{\mathrm{BKM}}(\beta) \equiv \chi_{A B}(\beta)=\int_{0}^{1} d \lambda K_{A B}(\beta ; \lambda)=\int_{-\infty}^{\infty} d \omega \frac{e^{\beta \hbar \omega}-1}{\beta \hbar \omega} J_{A B}(\omega ; \beta, 0),
$$

причем в высокотемпературном пределе $\beta \hbar \omega \ll 1$ имеем $K_{A B}^{\mathrm{BKM}}(\beta) \approx K_{A B}^{\mathrm{KMS}}(\beta)$.

Метрика БКМ была впервые получена Боголюбовым в работе [16] именно в форме правой части ${ }^{5)}(17)$ задолго до того, как в литературе появилось семейство метрик Вигнера-Яназе-Дайсона [12]. Заметим, что в целом подход Боголюбова-Тябликова [17] отличается от излагаемого здесь тем, что вместо вещественного “температурного" параметра $\lambda$ в нем использовался чисто мнимый "временно́й” параметр -it/ћ $\beta$ с заменой $e^{\lambda \beta \hbar \omega}$ на $e^{-i t \omega / \hbar}$ и $d / d \lambda$ на $-i d / d t$.

Благодаря этому $\lambda$-деформированный оператор фон Неймана $[\rho(\beta)]^{\lambda}=e^{-\lambda \beta H}$ переходит в обычный оператор динамической эволюции $U(t)=e^{-i t H / \hbar}$, а операторное выражение $A(\beta ; \lambda)$ из $(3)$ - в представление Гейзенберга для оператора $A(\beta ; 0)$. Соответственно в работе [17] вместо статической квантовой корреляционной функции $K_{A B}(\beta ; \lambda)$ рассматривался ее временно́й аналог - функция $K_{A B}(\beta ; t)$, где после дифференцирования по $t$ в статическом пределе всегда полагается $t=0$.

Ввиду полного совпадения (с точностью до указанной замены переменных) уравнений Блоха $(10)$ для $\rho(\beta)$ и динамических уравнений движения Гейзенберга для $U(t)$ становится вполне естественным возникновение одинаковой структуры ${ }^{6)}$ правых частей (11) и выражений для частотных моментов $\overline{\omega^{n}}, n=-1,0,1,2, \ldots$.

\footnotetext{
4) Наличие этого множителя обусловлено появлением при $\lambda \neq 0$ оператора $\rho(\beta)$ в качестве сомножителя под знаком следа не в одном, как при $\lambda=0$, а в двух местах.

5) Левая часть (17) в работе [16] не обсуждалась, поскольку логика введения Боголюбовым новой метрики базировалась на методе функций Грина (см. ниже замечание после формулы (19)).

6)Это совпадение известно под названием "правил сумм" и используется для контроля вычислений.
} 
Согласно подходу Боголюбова-Тябликова все существенные черты рассматриваемой физической системы содержатся в выражении

$$
J_{A B}(\omega ; \beta, 0)=\sum_{\nu, \mu} A_{\nu \mu} B_{\mu \nu} e^{-\beta E_{\nu}} \delta\left(E_{\nu}-E_{\mu}-\hbar \omega\right), \quad J_{A A}(\omega ; \beta, 0) \geqslant 0,
$$

которое представляет собой явную запись следа от произведения операторов $A$ и $B$, входящего в определения квантовых корреляторов (1)-(3). Здесь $E_{\nu}-$ вещественные собственные значения ${ }^{7)}$ эрмитова гамильтониана $H, A_{\nu \mu}$ и $B_{\mu \nu}$ - матричные элементы операторов $A$ и $B$ по точным собственным функциям $H$ (предполагается, что они существуют, образуя полный ортонормированный базис).

В частности, при $B=A$ имеем $A_{\nu \mu} B_{\mu \nu}=\left|A_{\nu \mu}\right|^{2}$, так что величина $J_{A A}(\omega ; \beta, 0)$ является вещественной и неотрицательной, что позволяет рассматривать ее как операторную норму (метрику). Функционал $J_{A B}(\omega ; \beta, 0)$ обладает всеми свойствами скалярного произведения, присущими $K_{A B}(\beta ; 0)$ : действительно, его комплексность и билинейность по операторным аргументам $A$ и $B$ очевидны из определения (18), а согласно Боголюбову [16] для (18) выполняется и важнейшее свойство скалярного произведения

$$
J_{B A}(\omega ; \beta, 0)=J_{A B}^{*}(\omega ; \beta, 0)=J_{A B}(-\omega ; \beta, 0) e^{-\beta \hbar \omega}
$$

Существенно, что присущие величине $J_{A B}(\omega ; \beta, 0)$ "хорошие" свойства не изменяются при умножении ее на любой строго положительный множитель, в общем случае зависящий от $\omega, \beta$ и $\lambda$; в частности, при $\lambda \neq 0$ имеет место небольшая модификация указанного свойства симметрии:

$$
J_{B A}(\omega ; \beta, \lambda)=J_{A B}^{*}(\omega ; \beta, \lambda)=J_{A B}(-\omega ; \beta, 1-\lambda) .
$$

Физический интерес представляют два случая, обобщающие операторную метрику $\left.J_{A A}(\omega ; \beta, 0) \equiv J_{A A}^{\mathrm{KMS}}(\omega ; \beta, 0): 1\right)$ случай метрики $J_{A A}^{\mathrm{WYD}}(\omega ; \beta, \lambda)$ при $\lambda \neq 0$, когда согласно (14) указанный множитель равен $\left.e^{\lambda \beta \hbar \omega} ; 2\right)$ случай метрики $J_{A A}^{\mathrm{BKM}}(\omega ; \beta)$, когда согласно (17) указанный множитель равен $\varphi(\omega ; \beta)=\left(e^{\beta \hbar \omega}-1\right) / \beta \hbar \omega ;$ именно этот результат был впервые получен и сформулирован Боголюбовым в работе [16].

Разумеется, точно вычислить величину (18) удается лишь относительно редко для простых модельных систем ${ }^{8)}$, однако в подходе Боголюбова-Тябликова имеется весьма эффективный способ вычисления $J_{A B}(\omega ; \beta, 0)$ (для сложных систем, разумеется, приближенного) посредством аппарата двухвременнь́іх температурных запаздывающих и опережающих функций Грина (см. [17], [21]-[23]).

Именно, имеет место замечательная спектральная теорема, впервые установленная в работе [17]:

$$
J_{A B}^{(\eta)}(\omega ; \beta, 0)\left(e^{\beta \hbar \omega}+\eta\right)=G_{A B}^{(\eta)}(\omega+i \varepsilon)-G_{A B}^{(\eta)}(\omega-i \varepsilon),
$$

7) Заметим, что при вырождении состояний, когда $E_{\nu}=E_{\mu}$, величина (18) имеет сингулярность $\delta(\omega)$.

${ }^{8)}$ В контексте данной работы в основном существенны свойства спектральной интенсивности (14) и (18), не требующие ее явного вычисления; пример такого вычисления будет приведен в разделе 5 . 
где $E$ - комплексный аргумент фурье-образа $G_{A B}^{(\eta)}(E)$ коммутаторной или антикоммутаторной функции Грина с $\eta=+1$ или $\eta=-1$ соответственно. Величина $G_{A B}^{(\eta)}(E)$ удовлетворяет цепочке уравнений движения

$$
E G_{A B}^{(\eta)}(E)=\frac{i}{2 \pi}\left\langle[A, B]_{\eta}\right\rangle+G_{C B}^{(\eta)}(E)
$$

где $C=[A, H]_{-}$и т.д.; кроме того, $G_{A B}^{(\eta)}(E)$ обладает спектральным представлением и просто связана с рассмотренным выше (конец раздела 2) статическим изотермическим откликом:

$$
G_{A B}^{(\eta)}(E)=\frac{1}{2 \pi} \int_{-\infty}^{\infty} d \omega J_{A B}^{(\eta)}(\omega ; \beta, 0) \frac{e^{\beta \hbar \omega}+\eta}{E-\omega}, \quad \delta\left\langle B^{\dagger}\right\rangle=-2 \pi G_{A B}^{(-)}(0) .
$$

Один из основных результатов $\S 5$ работы Боголюбова [16] состоит в том, что нулевые фурье-образы функций Грина $G_{A B}^{(-)}(0)$ удовлетворяют аксиоматике комплексного скалярного произведения на алгебре операторов $A, B$, а пропорциональность $G_{A B}^{(-)}(0)$ вариации среднего значения $\delta\left\langle B^{\dagger}\right\rangle$ позволяет просто перенести эту аксиоматику на восприимчивости $\chi_{A B}$ (см. конец раздела 2$)$.

\section{4. СВОЙСТВА $\lambda$-ДЕФОРМИРОВАННОЙ ОПЕРАТОРНОЙ НОРМЫ}

Положим $B=A$ в выражении (14) и рассмотрим подробнее свойства операторной нормы ${ }^{9)} K_{A A}(\beta ; \lambda) \geqslant 0$ и связанных с ней величин $(15)-(17)$. Используя свойство $(20)$ величины $J_{A A}(\omega ; \beta, \lambda) \geqslant 0$, для которой замена $\omega$ на $-\omega$ равносильна замене $\lambda$ на $1-\lambda$, перейдем в (14)-(17) к интегрированию только по положительной полуоси $\omega \geqslant 0$ :

$$
\begin{aligned}
K_{A A}(\beta ; \lambda) & =\int_{0}^{\infty} d \omega J_{A A}(\omega ; \beta, 0) \psi_{+}(\omega ; \beta, \lambda), \\
\left(\frac{1}{\beta}\right)^{2} K_{A A}^{\prime \prime}(\beta ; \lambda) & =\int_{0}^{\infty} d \omega \omega^{2} J_{A A}(\omega ; \beta, 0) \psi_{+}(\omega ; \beta, \lambda), \\
\frac{1}{\beta} K_{A A}^{\prime}(\beta ; \lambda) & =\int_{0}^{\infty} d \omega \omega J_{A A}(\omega ; \beta, 0) \psi_{-}(\omega ; \beta, \lambda), \\
\chi_{A A}(\beta) & =\int_{0}^{\infty} d \omega J_{A A}(\omega ; \beta, 0) \varphi_{-}(\omega ; \beta) .
\end{aligned}
$$

Функции $\psi_{ \pm}(\omega ; \beta, \lambda)$ и $\varphi_{ \pm}(\omega ; \beta)$ определены равенствами

$$
\psi_{ \pm}(\omega ; \beta, \lambda)=e^{\lambda \beta \hbar \omega} \pm e^{(1-\lambda) \beta \hbar \omega}, \quad \varphi_{ \pm}(\omega ; \beta)=\frac{e^{\beta \hbar \omega} \pm 1}{\beta \hbar \omega / 2}
$$

и удовлетворяют следующим полезным для дальнейшего соотношениям:

$$
\begin{gathered}
\frac{d}{d \lambda} \psi_{ \pm}(\omega ; \beta, \lambda)=\beta \hbar \omega \psi_{\mp}(\omega ; \beta, \lambda) \\
\int_{0}^{1} d \lambda \psi_{+}(\omega ; \beta, \lambda)=\varphi_{-}(\omega ; \beta), \quad \int_{0}^{1} d \lambda \psi_{-}(\omega ; \beta, \lambda)=0 .
\end{gathered}
$$

9)Здесь и далее в этом разделе речь идет об общем случае метрик $K_{A A}^{\mathrm{WYD}}(\beta ; \lambda)$. 
Часто удобна запись указанных функций с помощью переменной $\theta=\beta \hbar \omega / 2>0$ :

$$
\begin{gathered}
\varphi_{+}(\theta)=2 e^{\theta} \frac{\operatorname{ch} \theta}{\theta}, \quad \varphi_{-}(\theta)=2 e^{\theta} \frac{\operatorname{sh} \theta}{\theta}, \quad \frac{\varphi_{-}(\theta)}{\varphi_{+}(\theta)}=\operatorname{th} \theta \leqslant 1, \\
\psi_{+}(\theta ; \lambda)=2 e^{\theta} \operatorname{ch}\left[2 \theta\left(\frac{1}{2}-\lambda\right)\right], \quad \psi_{-}(\theta ; \lambda)=-2 e^{\theta} \operatorname{sh}\left[2 \theta\left(\frac{1}{2}-\lambda\right)\right] .
\end{gathered}
$$

Заметим, что функции $\varphi_{-}(\theta)$ и $\psi_{+}(\theta ; \lambda)$ всегда положительно знакоопределены при любых значениях $\theta$ и $\lambda$, а функции $\varphi_{+}(\theta)$ и $\psi_{-}(\theta ; \lambda)$ меняют знак вместе с $\theta$. Кроме того, функция $\psi_{+}(\theta ; \lambda)$ четна относительно замены $\lambda$ на $1-\lambda$, а функция $\psi_{-}(\theta ; \lambda)$ нечетна, причем $\psi_{-}(\theta ; \lambda)<0$, если $0 \leqslant \lambda<1 / 2$, или $\psi_{-}(\theta ; \lambda)>0$, если $1 / 2<\lambda \leqslant 1$, так что $\psi_{-}(\theta ; 1 / 2)=0$.

Указанные свойства позволяют достаточно полно описать поведение трех величин из (21) при всех значениях $\lambda$; положение $\chi_{A A}(\beta)$ в семействе $K_{A A}(\beta ; \lambda)$ будет далее рассмотрено отдельно.

Первое из соотношений (21) подтверждает полученный ранее из общих соображений вывод (см. формулы (4) и (5)): все метрики $K_{A A}(\beta ; \lambda)$, образующие семейство ВЯД, действительно являются строго положительными, обладают свойством симметрии $K_{A A}(\beta ; \lambda)=K_{A A}(\beta ; 1-\lambda)$ и для них справедливы утверждения, сформулированные после формулы $(6)$.

Отметим, что в формальном пределе бесконечной температуры $\beta=0$ все метрики $K_{A A}(\beta ; \lambda)$ становятся одинаковыми и равными предельному значению

$$
K_{A A}(0)=2 \int d \omega J_{A A}(\omega ; 0,0)
$$

не зависящему от $\lambda$ (интегрирование проводится по $\omega \geqslant 0)$.

Второе и третье из соотношений (21) описывают свойства гладкости $K_{A A}(\beta ; \lambda)$ как функции параметра деформации $\lambda$, которые не могли быть получены из общих соображений симметрии (4) и (5), поскольку для этого необходимо и целесообразно использование спектральных представлений (14). Из второго соотношения (21) следует свойство выпуклости вниз функции $K_{A A}(\beta ; \lambda)$, поскольку $\left(d^{2} / d \lambda^{2}\right) K_{A A}(\beta ; \lambda)>$ 0 при всех значениях $\lambda$; как видно из сравнения первого и второго соотношений $(21)$, зависимость от $\lambda$ второй производной функции $K_{A A}(\beta ; \lambda)$ повторяет поведение самой этой функции. Из третьего соотношения (21) следует монотонное убывание функции $K_{A A}(\beta ; \lambda) \geqslant 0$ на отрезке $0 \leqslant \lambda<1 / 2$ от максимума $K_{A A}^{\mathrm{KMS}}(\beta ; 0)$ до минимума $K_{A A}^{\mathrm{WY}}(\beta ; 1 / 2)$ : на этом отрезке производная $(d / d \lambda) K_{A A}(\beta ; \lambda)$ отрицательна, но монотонно возрастает до нуля ${ }^{10)}$.

Учитывая, что максимальное и минимальное значения функции $\psi_{+}(\omega ; \beta, \lambda)$ равны соответственно $\psi_{+}(\omega ; \beta, 0)=1+e^{\beta \hbar \omega}=2 e^{\theta} \operatorname{ch} \theta$ и $\psi_{+}(\omega ; \beta, 1 / 2)=2 e^{\beta \hbar \omega / 2}=2 e^{\theta}$, имеем

$$
\begin{aligned}
K_{A A}^{\mathrm{KMS}}(\beta ; 0) & =\int_{0}^{\infty} d \omega J_{A A}(\omega ; \beta, 0)\left(1+e^{\beta \hbar \omega}\right), \\
K_{A A}^{\mathrm{WY}}(\beta ; 1 / 2) & =2 \int_{0}^{\infty} d \omega J_{A A}(\omega ; \beta, 0) e^{\beta \hbar \omega / 2} .
\end{aligned}
$$

10) Указанное свойство в литературе иногда называют "сжатием метрики". 
Как и следовало ожидать, глобальная, или интегральная, метрика БКМ $K_{A A}^{\mathrm{BKM}}(\beta)$ занимает промежуточное место между экстремальными метриками (24), которое регулируется всегда положительным отношением ${ }^{11)} \varphi_{-}(\theta) / \psi_{+}(\theta ; \lambda)$. Это следует из простых оценок $\psi_{+}(\theta ; 1 / 2) \leqslant \varphi_{-}(\theta) \leqslant \psi_{+}(\theta ; 0)$, или в явном виде $\operatorname{cth} \theta \geqslant 1 / \theta \geqslant 1 / \operatorname{sh} \theta$, что равносильно паре неравенств $\theta \geqslant \operatorname{th} \theta$ и $\theta \leqslant \operatorname{sh} \theta$. Эти неравенства обращаются в равенства лишь в предельном случае очень высоких температур $\theta=0$.

Нетрудно показать и более точно, что при всех температурах $\theta>0$ значение $K_{A A}^{\mathrm{BKM}}(\beta)$ находится ближе к $K_{A A}^{\mathrm{WY}}(\beta ; 1 / 2)$, чем к $K_{A A}^{\mathrm{KMS}}(\beta ; 0)$. Составим для этого две заведомо положительные разности

$$
\Delta_{1}(\beta)=K_{A A}^{\mathrm{KMS}}(\beta ; 0)-K_{A A}^{\mathrm{BKM}}(\beta), \quad \Delta_{2}(\beta)=K_{A A}^{\mathrm{BKM}}(\beta)-K_{A A}^{\mathrm{WY}}(\beta ; 1 / 2)
$$

и покажем, что $\Delta_{1}(\beta)-\Delta_{2}(\beta) \geqslant 0$. Как видно из соотношений $(24)$ и последнего из равенств $(21)$, величины $\Delta_{1}(\theta)$ и $\Delta_{2}(\theta)$ определяются соответственно двумя положительными разностями:

$$
\begin{gathered}
\Delta_{1}(\theta)=\psi_{+}(\theta ; 0)-\varphi_{-}(\theta)=\left(1+e^{2 \theta}\right)\left(1-\frac{\operatorname{th} \theta}{\theta}\right), \\
\Delta_{2}(\theta)=\varphi_{-}(\theta)-\psi_{+}(\theta ; 1 / 2)=2 e^{\theta}\left(\frac{\operatorname{sh} \theta}{\theta}-1\right) .
\end{gathered}
$$

Тогда искомая разность $\Delta_{1}(\theta)-\Delta_{2}(\theta)$ определяется всегда положительной величиной

$$
\psi_{+}(\theta ; 0)-2 \varphi_{-}(\theta)+\psi_{+}(\theta ; 1 / 2)=\left(1+e^{\theta}\right)^{2}\left(1-\frac{\operatorname{th} \theta}{\theta}\right)+2 e^{\theta} \frac{\operatorname{th} \theta}{\theta}(\operatorname{ch} \theta-1) .
$$

Еще одна физически интересная величина, а именно разность максимальной и минимальной метрик $K_{A A}^{\mathrm{KMS}}(\beta ; 0)-K_{A A}^{\mathrm{WY}}(\beta ; 1 / 2)$, определяется суммой $\Delta_{1}(\theta)+\Delta_{2}(\theta)$ :

$$
\Delta_{1}(\theta)+\Delta_{2}(\theta)=\psi_{+}(\theta ; 0)-\psi_{+}(\theta ; 1 / 2)=\left(1-e^{\theta}\right)^{2} \geqslant 0,
$$

также положительной при всех $\theta>0$, т.е. при всех температурах $T=1 / k_{\mathrm{B}} \beta>0$.

Однако знания указанных выше свойств операторной нормы $K_{A A}(\beta ; \lambda)$ еще недостаточно для того, чтобы вычислить представляющую физический интерес КБШ-разность $\Delta_{A B}(\beta ; \lambda) \geqslant 0$, определенную выражением (6). Для этого необходимо знать также зависимость от $\lambda$ скалярного произведения $K_{A B}(\beta ; \lambda)$, которую нетрудно найти, вновь перейдя к интегрированию только по положительным частотам.

С учетом свойств (16) вещественная и мнимая части $J_{A B}(\omega ; \beta, 0)$ имеют вид

$$
\begin{aligned}
& \operatorname{Re} J_{A B}(\omega ; \beta, 0)=\frac{1}{2} J_{A B}^{(+)}(\omega ; \beta, 0), \quad \operatorname{Im} J_{A B}(\omega ; \beta, 0)=\frac{1}{2 i} J_{A B}^{(-)}(\omega ; \beta, 0) \\
& J_{A B}^{( \pm)}(\omega ; \beta, 0) \equiv J_{A B}(\omega ; \beta, 0) \pm J_{A B}^{*}(\omega ; \beta, 0)=J_{A B}(\omega ; \beta, 0) \pm J_{B A}(\omega ; \beta, 0)
\end{aligned}
$$

11)Это возможно благодаря всегда положительному множителю $J_{A A}(\omega ; \beta, 0)$ во всех интегралах в равенствах (21). 
Тогда $\lambda$-деформированное скалярное произведение можно записать в виде

$$
\begin{aligned}
& K_{A B}(\beta ; \lambda)=K_{A B}^{(+)}(\beta ; \lambda)+i K_{A B}^{(-)}(\beta ; \lambda) \\
& K_{A B}^{( \pm)}(\beta ; \lambda)=\int_{0}^{\infty} d \omega J_{A B}^{( \pm)}(\omega ; \beta, 0) \psi_{ \pm}(\omega ; \beta, \lambda)
\end{aligned}
$$

где величины $K_{A B}^{( \pm)}(\beta ; \lambda)$, определенные в $(7)$, входят в КБШ-разность в виде (8). Аналоги величин $K_{A B}^{( \pm)}(\beta ; \lambda)$ в метрике БКМ имеют вид

$$
\chi_{A B}^{(+)}(\beta)=\int_{0}^{1} d \lambda K_{A B}^{(+)}(\beta ; \lambda)=\int_{0}^{\infty} d \omega J_{A B}^{(+)}(\omega ; \beta, 0) \varphi_{-}(\omega ; \beta), \quad \chi_{A B}^{(-)}(\beta) \equiv 0,
$$

где использованы второе и третье из соотношений (23). Существенно, что в общем случае ненулевой вклад в недиагональную восприимчивость в метрике БКМ может давать только вещественная часть спектральной интенсивности $J_{A B}^{(+)}(\omega ; \beta, 0)$.

Несмотря на внешнее сходство выражений (27) и (28) с выражениями (21), положительно определенное отношение $\varphi_{-}(\omega ; \beta) / \psi_{+}(\omega ; \beta, \lambda)$ уже не определяет (как это было в $(26))$ положение восприимчивости $\chi_{A B}^{(+)}(\beta)$ относительно недиагонального коррелятора $K_{A B}^{(+)}(\beta ; \lambda)$. Это различие связано с отсутствием знакоопределенности величины $J_{A B}^{( \pm)}(\omega ; \beta, 0)$ в отличие от $J_{A A}(\omega ; \beta, 0)$ (см. сноску 11$)$.

Аналогично относительное положение тождественно равной нулю восприимчивости $\chi_{A B}^{(-)}(\beta)$ и коррелятора $K_{A B}^{(-)}(\beta ; \lambda)$ не может быть определено однозначно, поскольку оно зависит от знаков входящих в $(27)$ функций $\psi_{-}(\omega ; \beta, \lambda)$ и $J_{A B}^{(+)}(\omega ; \beta, 0)$, причем ни одна из них в общем случае не является знакоопределенной.

Минимизация КБШ-разности $\Delta_{A B}(\beta ; \lambda)$ могла бы, на первый взгляд, достигаться при использовании метрики $K_{A A}^{\mathrm{WY}}(\beta ; 1 / 2)$, минимизирующей всегда положительное произведение $K_{A A}(\beta ; \lambda) K_{B B}(\beta ; \lambda)$, или уменьшаемое $\Delta_{A B}(\beta ; \lambda)$. Однако нет никаких оснований считать, что при этом то же самое значение $\lambda=1 / 2$ максимизирует также всегда положительную величину $\left|K_{A B}(\beta ; \lambda)\right|^{2}$, или вычитаемое $\Delta_{A B}(\beta ; \lambda)$. Более того, как будет показано ниже, для $\left|K_{A B}(\beta ; \lambda)\right|^{2}$ имеет место как раз обратное поведение.

Рассмотрим практически важный случай, когда спектральная интенсивность оказывается чисто мнимой ${ }^{12)}$, так что $J_{A B}^{(+)}(\omega ; \beta, 0)=0$ и $K_{A B}^{(+)}(\beta ; \lambda)=0$. В этом случае вклад в вычитаемое КБШ-разности для семейства ВЯД определяется только величиной $\left|K_{A B}^{(-)}(\beta ; \lambda)\right|^{2}$, неотрицательной при всех значениях $\lambda$, но в силу свойства $\psi_{-}(\omega ; \beta, 1 / 2)=0$ имеющей при $\lambda=1 / 2$ абсолютный минимум, равный нулю. При этом, как было показано выше, обе величины, входящие в произведение $K_{A A}(\beta ; \lambda) K_{B B}(\beta ; \lambda)$ (уменьшаемое КБШ-разности), в силу свойств функции $\psi_{+}(\omega ; \beta, \lambda)$ также достигают абсолютного минимума (отличного от нуля) в той же точке $\lambda=1 / 2$. Для метрики БКМ в рассматриваемом случае равны нулю обе величины $\chi_{A B}^{( \pm)}(\beta)$, так что КБШ-разность содержит только уменьшаемое $\chi_{A A}(\beta) \chi_{B B}(\beta)$.

${ }^{12)}$ Согласно спектральной теореме (19) это соответствует наличию у $G_{A B}(E)$ только вещественных полюсов; см. ниже пример в разделе 5 . 
Таким образом, априори нельзя утверждать, что значение $\lambda=1 / 2$ (и, следовательно, "минимальная" метрика ВЯ) или какое-либо другое фиксированное значение $\lambda$ (или даже метрика БКМ) доставляет минимум значению КБШ-разности для любой физической системы и любой пары операторов $A$ и $B$.

Более того, как показывает простой модельный пример (раздел 5), возможны случаи, когда КБШ-разность для всего семейства метрик ВЯД вообще не зависит от $\lambda$ и при этом не превышает КБШ-разности в метрике БКМ.

\section{5. ПРИМЕР: ИДЕАЛЬНЫЙ СПИНОВЫЙ ПАРАМАГНЕТИК И АЛГЕБРА НАБЛЮДАЕМЫХ $S U(2)$}

Рассмотрим идеальный газ $N$ спинов, обладающих магнитным моментом $\mu=g \mu_{\mathrm{B}}$ $\left(g\right.$ - фактор Ланде, $\mu_{\mathrm{B}}$ - магнетон Бора) и находящихся в постоянном магнитном поле $h$, направленном вдоль оси $z$; при этом газ помещен в термостат с обратной температурой $\beta>0$. В этом случае наблюдаемые образуют алгебру $S U(2)$, включающую три независимых генератора $\left\{S_{x}, S_{y}, S_{z}\right\}$ с перестановочными соотношениями $\left[S_{x}, S_{y}\right]_{-}=i S_{z}$ и т.д. (циклические перестановки координат $\left.x, y, z\right)$. Оператор Казимира имеет вид $S_{x}^{2}+S_{y}^{2}+S_{z}^{2}=S(S+1)$, где $S \geqslant 1 / 2-$ спиновое квантовое число (целое или полуцелое), а гамильтониан системы (в расчете на один спин) соответствует учету энергии Зеемана спина в поле и равен $H / N=-\hbar \omega_{0} S_{z}$, где $\hbar \omega_{0}=\mu h$.

Рассмотрим обобщенное СН, или КБШ-разность, для пары операторов $S_{x}$ и $S_{y}$, которые не являются интегралами движения, так что построенные из них $\lambda$-деформированные скалярные произведения $K_{\alpha \beta}(\beta ; \lambda) \equiv\left\langle S_{\alpha}(\lambda) S_{\beta}\right\rangle, \alpha, \beta=x, y$, зависят от параметра $\lambda$. Для получения КБШ-разности необходимо, используя формулы (21) и $(25)$, найти два диагональных коррелятора $K_{\alpha \alpha}(\beta ; \lambda)$, равных друг другу в силу аксиальной симметрии системы, а также недиагональный коррелятор $K_{\alpha \beta}^{( \pm)}(\beta ; \lambda)$, $\alpha \neq \beta$.

Следуя описанной в разделе 4 схеме, найдем соответствующие функции Грина $G_{x x}(E)$ и $G_{x y}(E)$ и спектральные интенсивности $J_{x x}(\omega)$ и $J_{x y}(\omega)$, используя простые уравнения движения для операторов

$$
i \hbar \frac{d S_{x, y}}{d t}=\left[S_{x, y}, H\right]_{-}= \pm i \hbar \omega_{0} S_{y, x}
$$

Здесь удобны коммутаторные функции Грина с $\eta=-1$, для которых имеет место система двух линейных уравнений

$$
E G_{x y}(E)=-\frac{1}{2 \pi} \sigma(\beta)+i \hbar \omega_{0} G_{y y}(E), \quad E G_{y y}(E)=-i \hbar \omega_{0} G_{x y}(E),
$$

решения которой имеют вид суперпозиций двух полюсных членов вида $\left(E \pm \hbar \omega_{0}\right)^{-1}$.

Учитывая, что $(x \pm i \varepsilon)^{-1}=P(1 / x) \mp i \pi \delta(x)$, найдем с помощью спектральной теоремы (20) следующие выражения для спектральных интенсивностей:

$$
\begin{aligned}
& J_{x x}(\omega ; \beta, 0)=J_{y y}(\omega ; \beta, 0)=\frac{1}{2} \sigma(\beta)\left[\delta\left(\omega-\omega_{0}\right)-\delta\left(\omega+\omega_{0}\right)\right]\left(e^{\beta \hbar \omega}-1\right)^{-1}, \\
& J_{x y}(\omega ; \beta, 0)=J_{y x}^{*}(\omega ; \beta, 0)=\frac{i}{2} \sigma(\beta)\left[\delta\left(\omega-\omega_{0}\right)+\delta\left(\omega+\omega_{0}\right)\right]\left(e^{\beta \hbar \omega}-1\right)^{-1} .
\end{aligned}
$$


Здесь $\sigma(\beta)=(1 / i)\left\langle\left[S_{x}, S_{y}\right]_{-}\right\rangle=\left\langle S_{z}\right\rangle$, причем $\sigma(\beta) \geqslant 0$ (явное выражение см. ниже в конце данного раздела). Очевидно, что в силу симметрии $\left\langle S_{x}\right\rangle=\left\langle S_{y}\right\rangle$, причем $\left\langle S_{x}\right\rangle=\left\langle S_{y}\right\rangle=0$ с учетом свойства $\left[S_{x, y}, H\right]_{-} \neq 0$. Как видно из $(29), J_{x y}(\omega ; \beta, 0)$ содержит только мнимую часть, так что только величина $J_{x y}^{(-)}(\omega ; \beta, 0)$, а тем самым и $K_{x y}^{(-)}(\beta ; \lambda)$, отлична от нуля, тогда как $K_{x y}^{(+)}(\beta ; \lambda) \equiv 0$. В этом случае согласно $(28)$ в метрике БКМ равна нулю не только восприимчивость $\chi_{x y}^{(-)}(\beta)$ (как всегда), но и $\chi_{x y}^{(+)}(\beta) \equiv 0$, так что

$$
\chi_{x y}(\beta)=\chi_{x y}^{(+)}(\beta)+i \chi_{x y}^{(-)}(\beta) \equiv 0 .
$$

Полагая, как и ранее, $\theta=\beta \hbar \omega_{0} / 2(\theta \geqslant 0)$, согласно формулам $(21),(27)$ и (29) находим диагональные $(\alpha=\beta=x, y)$ и недиагональные $(\alpha \neq \beta)$ корреляторы $K_{\alpha \beta}^{\mathrm{WYD}}(\beta ; \lambda)$ :

$$
\begin{aligned}
& K_{x y}^{(-)}(\beta ; \lambda)=-\sigma(\beta) \psi_{-}\left(\omega_{0} ; \beta, \lambda\right)\left(e^{\beta \hbar \omega_{0}}-1\right)^{-1}=\sigma(\theta) \frac{\operatorname{sh}[2 \theta(1 / 2-\lambda)]}{\operatorname{sh} \theta}, \\
& K_{x x}(\beta ; \lambda)=K_{y y}(\beta ; \lambda)=\sigma(\beta) \psi_{+}\left(\omega_{0} ; \beta, \lambda\right)\left(e^{\beta \hbar \omega_{0}}-1\right)^{-1}=\sigma(\theta) \frac{\operatorname{ch}[2 \theta(1 / 2-\lambda)]}{\operatorname{sh} \theta},
\end{aligned}
$$

а также диагональные и недиагональные обобщенные восприимчивости $K_{\alpha \beta}^{\mathrm{BKM}}(\beta) \equiv$ $\chi_{\alpha \beta}(\beta)$ :

$$
\begin{aligned}
& \chi_{x x}(\theta)=\chi_{y y}(\theta)=\sigma(\beta) \varphi_{-}\left(\omega_{0} ; \beta\right)\left(e^{\beta \hbar \omega_{0}}-1\right)^{-1}=\frac{\sigma(\theta)}{\theta} \\
& \chi_{x y}(\theta)=\chi_{y x}(\theta)=0
\end{aligned}
$$

В соответствии с общими результатами (раздел 4, после формулы (24)) для диагональных корреляторов и восприимчивостей имеем неравенства

$$
\begin{gathered}
K_{x x}\left(\theta ; \frac{1}{2}\right) \leqslant \chi_{x x}(\theta) \leqslant K_{x x}(\theta ; 0) \\
K_{x x}(\theta ; 0) \equiv K_{x x}^{\mathrm{KMS}}(\theta)=\sigma(\theta) \operatorname{cth} \theta, \quad K_{x x}\left(\theta ; \frac{1}{2}\right)=K_{x x}^{\mathrm{WY}}(\theta)=\frac{\sigma(\theta)}{\operatorname{sh} \theta},
\end{gathered}
$$

а для недиагональных -

$$
\begin{gathered}
0=K_{x y}\left(\theta ; \frac{1}{2}\right)=\chi_{x y}(\theta) \leqslant K_{x y}(\theta ; 0) \\
K_{x y}(\theta ; 0)=K_{x y}^{\mathrm{KMS}}(\theta)=\sigma(\theta), \quad K_{x y}\left(\theta ; \frac{1}{2}\right)=K_{x y}^{\mathrm{WY}}(\theta)=0 .
\end{gathered}
$$

Видно, что в обоих случаях БКМ-восприимчивость не превышает значения КМШ-коррелятора, а равенство достигается лишь в высокотемпературном пределе $\theta=0$; интересно, что $\chi_{x y}(\theta)$ уже не просто близок к $K_{x y}^{\mathrm{WY}}(\theta)$, как это имеет место для их диагональных аналогов $\chi_{x x}(\theta)$ и $K_{x x}^{\mathrm{WY}}(\theta)$, но и полностью совпадает с ним.

Искомая КБШ-разность

$$
\Delta_{x y}(\beta ; \lambda)=K_{x x}(\beta ; \lambda) K_{y y}(\beta ; \lambda)-\left|K_{x y}^{(-)}(\beta ; \lambda)\right|^{2}
$$


для $\lambda$-семейства ВЯД оказывается равной $[\sigma(\theta) / \operatorname{sh} \theta]^{2}$, где учтены выражения (30) и тождество $\operatorname{ch}^{2} x-\operatorname{sh}^{2} x \equiv 1$. Таким образом, вопреки ожиданиям, $\Delta_{x y}(\beta ; \lambda)$ в данной модели вообще не зависит от параметра деформации $\lambda$ и просто совпадает со своим значением в исходной метрике КМШ при $\lambda=0$.

С учетом (31) КБШ-разность в метрике БКМ равна $\Delta_{x y}(\theta)=[\sigma(\theta) / \theta]^{2}$, так что отношение $\Delta_{x y}^{\mathrm{BKM}}(\theta) / \Delta_{x y}^{\mathrm{WYD}}(\theta)=[\operatorname{sh} \theta / \theta]^{2} \geqslant 1$ при всех $\theta$ (знак равенства достигается только при $\theta=0$ ). Этот результат означает невозможность понижения значения КБШ-разности и, следовательно, минимизации обобщенного СН в форме Шредингера для любой пары некоммутирующих наблюдаемых в метрике БКМ по сравнению с метрикой КМШ (а в данном случае и для всего семейства метрик ВЯД).

По-видимому, физически это соответствует большей роли тепловых флуктуаций в формировании БКМ-восприимчивостей по сравнению с ВЯД-корреляторами. Разумеется, степень общности этого утверждения следовало бы проверить и на других, не столь простых, спиновых моделях (например, на модели Изинга).

Рассмотрим в заключение поведение КБШ-разности $\Delta_{x y}(\theta)$ в любой из метрик в предельных случаях низких $(T \rightarrow 0, \theta \rightarrow \infty)$ и высоких $(T \rightarrow \infty, \theta \rightarrow 0)$ температур. Здесь принимается, что $h=$ const; учитывая, что переменная $\theta=\left(\hbar / 2 k_{\mathrm{B}}\right)(\mu h / T)$, аналогичный расчет для КБШ-разности может быть проведен также и при фиксированной температуре $T=$ const для случаев высокополевого предела, когда $h \rightarrow \infty$, $\theta \rightarrow \infty$, и низкополевого предела, когда $h \rightarrow 0, \theta \rightarrow 0$.

Уже на качественном уровне ясно, что при низких температурах $\sigma(\theta) \rightarrow \sigma_{0}>0$, так что $\Delta_{x y} \rightarrow 0$, т.е. при $T=0$ для идеального спинового парамагнетика всегда имеет место насыщение СН Шредингера (для метрик ВЯД и БКМ будет различен лишь темп этого стремления по переменной $\theta)$.

В высокотемпературном (квазиклассическом) пределе ситуация несколько сложнее, поскольку при $\theta \rightarrow 0$ не только $\operatorname{sh} \theta \rightarrow 0$, но также $\sigma(\theta) \rightarrow 0$, так что для $\Delta_{x y}(\theta)$ возникает неопределенность типа $0 / 0$; таким образом, необходимо располагать явным выражением для $\sigma(\theta)$ и его асимптотики при $\theta \rightarrow 0$.

Элементарный расчет по формуле $(1)$, где $A=S_{z}, \rho(\theta)=(1 / Z(\theta)) e^{2 \theta S_{z}}, Z(\theta)=$ $\operatorname{Sp} e^{2 \theta s_{z}},-S \leqslant S_{z} \leqslant S$, приводит к выражению для $\sigma(\theta)=(1 / 2) d \ln Z(\theta) / d \theta$ через известную в квантовой теории магнетизма функцию Бриллюэна $B_{S}(\theta)$ :

$$
\frac{\sigma(\theta)}{\sigma_{0}}=B_{S}(\theta)=\frac{1}{2 S}[(2 S+1) \operatorname{cth}(2 S+1) \theta-\operatorname{cth} \theta], \quad B_{S}(\infty)=1, \quad \sigma_{0}=2 S .
$$

В пределе $\theta \rightarrow 0$ имеют место разложения $\operatorname{cth}(a \theta) \approx 1 / a \theta+a \theta / 3+\cdots, a>0$, и $\operatorname{sh} \theta \approx \theta$, причем расходящиеся слагаемые $O(1 / \theta)$ в (32) точно компенсируются, так что в этом пределе из $(32)$ следует $\sigma(\theta) \approx f(S) \theta$, откуда $\Delta_{x y}(\theta) \rightarrow f(S)$, где $f(S)=(4 / 3) S(S+1)>0$.

Таким образом, в высокотемпературном пределе ни в одной из метрик не достигается насыщение $\mathrm{CH}$ для поперечных спиновых компонент $S_{x}$ и $S_{y}$ спинового парамагнетика, находящегося в продольном внешнем поле. Это может означать, что вклад тепловых (классических) флуктуаций в этом пределе превышает вклад квантовых флуктуаций в низкотемпературном пределе (в основном состоянии). Этот 
вывод подтверждается тем, что $\Delta_{x y}(\theta)$ растет с ростом спинового квантового числа $S$, т.е. с возрастанием степени классичности рассматриваемой парамагнитной системы.

\section{6. ЗАКЛЮЧЕНИЕ}

Целью настоящей работы являлась демонстрация важной роли, которую играет в квантовой статистической механике операторная метрика в алгебре физических наблюдаемых, введенная Боголюбовым в 1961 г. в замечательной работе [16]. Эта метрика, сформулированная в терминах нулевых фурье-образов функций Грина, позволила Боголюбову в той же работе изящно и просто получить свои знаменитые неравенства для корреляторов, что повлекло появление значительного количества работ (см., например, [23]). В дальнейшем оказалось, что метрика БКМ является глобальной, или интегральной, характеристикой однопараметрического семейства метрик ВЯД.

В настоящей работе проведен систематический анализ как диагональных, так и недиагональных корреляционных функций в зависимости от параметра "деформации" стандартной формы скалярного произведения КМШ, известной в функциональном анализе как метрика Гильберта-Шмидта. Одновременно рассмотрены также обобщенные изотермические восприимчивости, соответствующие метрике БКМ, и получены неравенства между указанными видами диагональных корреляторов.

При проведении анализа широко использованы спектральные представления, введенные Кэлленом и Вельтоном [20], но получившие истинное теоретическое значение после создания Боголюбовым и Тябликовым [17] метода двухвременных температурных запаздывающих и опережающих функций Грина, позволяющих обойти прямое вычисление матричных элементов операторов.

В работе показано, что в общем случае корреляторы в метрике БКМ занимают промежуточное положение между корреляторами в экстремальных метриках и обладают ясным физическим смыслом обобщенной изотермической восприимчивости.

В качестве иллюстрации подробно рассмотрен пример для алгебры наблюдаемых $S U(2)$ в простейшей модели идеального квантового спинового парамагнетика. В этой модели и диагональные, и недиагональные функции Грина пар спиновых операторов, не являющихся интегралами движения и не коммутирующих друг с другом, обладают двумя симметричными простыми полюсами, а соответствующие им спектральные интенсивности - дельта-образными пиками одного или противоположных знаков. Основной вывод из рассмотрения этого примера состоит в том, что обобщенное СН в форме Шредингера (известное в функциональном анализе как неравенство КБШ) может вообще не зависеть от параметра деформации, причем его значение в метрике БКМ мажорирует соответствующее значение в метрике ВЯД.

Обобщение метрики БКМ на случай бесконечных систем и неограниченных операторов в рамках подхода $C^{*}$-алгебр было дано Харрисоном и Уонгом [24]. Рёпшторф [25] применил норму БКМ, обладающую логарифмической выпуклостью [15], для анализа магнитной восприимчивости в проблеме Кондо.

Метрика БКМ оказалась весьма полезной для развития так называемого метода параметрического оценивания в квантовой статистической механике, сформулированного в работах Хелстрома [26] и Холево [27]. В дальнейшем на этой основе 
в некоммутативной теории вероятностей и математической статистике возникло направление, известное как геометростатистика (термин Колмогорова). Оно было начато Ченцовым [28] и Амари [29] и развивалось в работах [30]-[34].

В рамках метода параметрического оценивания вычисляется верхний предел точности определения $c$-численных параметров матрицы плотности, или, что эквивалентно, минимальная величина флуктуаций этих параметров ${ }^{13)}$. В классической математической статистике искомая граница устанавливается теоремой Фишера-Крамера-Рао с однозначным заданием матрицы Фишера, составленной из логарифмических производных оцениваемого вероятностного распределения. В геометростатистике матрица Фишера имеет смысл метрики в пространстве Римана параметров этого распределения. В классической статистической механике с каноническим (экспоненциальным) распределением матрица Фишера совпадает с матрицей обобщенных восприимчивостей по соответствующим параметрам и, следовательно, совпадает с корреляционной матрицей величин, термодинамически сопряженных оцениваемым параметрам. Так, в простейшем случае одномерного канонического распределения (пары $\beta$ и $H$ ) неравенство Фишера-Крамера-Рао имеет смысл так называемого термодинамического СН Гиббса-Эйнштейна, поскольку матрица Фишера это дисперсия энергии, а оцениваемая точность - дисперсия обратной температуры. В этом случае указанное неравенство оказывается насыщенным. Однако для некоммутативного аналога этого неравенства выбор матрицы Фишера становится неоднозначным. В частности, Петц и Тот [33] и Стритер [19] считают наилучшим выбором обобщенную восприимчивость в форме БКМ (более детальное обсуждение этих вопросов можно найти в работе [35]).

Заметим, что в работах [30]-[33] был введен и описан класс монотонных (сжимающих) метрик, значительно более общий по сравнению с рассмотренным здесь семейством метрик ВЯД и заслуживающий отдельного анализа с использованием аналога глобальной метрики БКМ, спектральных представлений и метода функций Грина в формулировке Боголюбова-Тябликова.

\section{Список литературы}

[1] Н. Н. Боголюбов, А. А. Логунов, А. И. Оксак, И. Т. Тодоров, Основы аксиоматического подхода в квантовой теории поля, Наука, М., 1969.

[2] Ж. Эмх, Алгебраические методы в статистической механике и квантовой теории поля, Мир, М., 1976.

[3] У. Браттели, Д. Робинсон, Операторные алгебры и квантовая статистическая механика, Мир, М., 1982.

[4] Р. Рихтмайер, Приниипь современной математической физики, Т. 1, Мир, М., 1982.

[5] И. фон Нейман, Математические основы квантовой механики, Наука, М., 1964.

[6] С. Г. Крейн (ред.), Функиионалъный анализ, Справочно-математическая библиотека, Наука, М., 1972.

[7] П.А. М. Дирак, Принципы квантовой механики, Физматгиз, М., 1960.

[8] Н.Н. Боголюбов, “Лекции по квантовой статистике”, Собрание научных трудов в 12 томах, 6, Наука, М., 2006, 9-235.

13) Ту же роль, но по отношению к операторам наблюдаемых, играет рассмотренная нами КБШ-разность, или обобщенное СН в форме Шредингера. 
[9] Л.Д. Фаддеев, О.А. Якубовский, Лекции по квантовой механике для студентов-математиков, Изд-во ЛГУ, Л., 1980.

[10] R. Kubo, J. Phys. Soc. Japan, 12:6 (1957), 570-602; Вопросы квантовой теории необратимых процессов, Проблемы физики, ред. В. Л. Бонч-Бруевич, ИЛ, М., 1961, 39-72.

[11] P. C. Martin, J. Schwinger, Phys. Rev., 115:6 (1959), 1342-1373.

[12] E. P. Wigner, M. M. Yanase, Proc. Natl. Acad. Sci. USA, 49 (1963), 910-918.

[13] E. H. Lieb, Adv. Math., 11:3 (1973), 267-288.

[14] F. Bauman, R. Jost, "Remarks on a conjecture of Robinson and Ruelle concerning the quantum mechanical entropy", Проблемы теоретической физики ( $к$ 60-летию акад. Н. Н. Боголюбова), ред. Д. И. Блохинцев, Наука, М., 1969, 285-293.

[15] J. M. Luttinger, Progr. Theoret. Phys., Suppl. No. 37-38 (1966), 35-45.

[16] Н.Н.Боголюбов, "Квазисредние в задачах статистической механики", Собрание научных трудов в 12 томах, 6, Наука, М., 2006, 236-327; Н. Н. Боголюбов, Квазисредние в задачах статистической механики, Препринт Д-781, ОИЯИ, Дубна, 1961; Избранные труды по статистической физике, МГУ, М., 1979, 193-269.

[17] Н.Н.Боголюбов, С. В. Тябликов, ДАН СССР, 126 (1959), 53-56.

[18] H. Mori, Progr. Theoret. Phys., 33:3 (1965), 423-455.

[19] R. F. Streater, "The Information Manifold for Relatively Bounded Potentials", Проблемь современной математической физики, Сб. статей К 90-летию со дня рождения академика Н. Н. Боголюбова, Тр. МИАН, 228, Наука, М., 2000, 217-235.

[20] H. B. Callen, T. A. Welton, Phys. Rev., 83:1 (1951), 34-40.

[21] В. Л. Бонч-Бруевич, С. В. Тябликов, Метод функиий Грина в статистической механике, Физматлит, М., 1961.

[22] Д. Н. Зубарев, Ю. А. Церковников, "Метод двухвременных температурных функций Грина в равновесной и неравновесной статистической механике", Теоретическая и математическая физика, Тр. МИАН, 175, Наука, М., 1986, 134-177.

[23] Д. Рюэль, Статистическая механика. Строгие результаты, Мир, М., 1971.

[24] J. C. Garrison, J. Wong, Comm. Math. Phys., 26:1 (1972), 1-5.

[25] G. Roepstorff, Comm. Math. Phys., 46:3 (1976), 253-262.

[26] К. Хелстром, Квантовая проверка гипотез и оценивания, Мир, М., 1979.

[27] А.С. Холево, Вероятностные и статистические аспекты квантовой теории, ИКИ, Москва-Ижевск, 2003.

[28] Н.Н. Ченцов, Статистические решающие правила и оптимальные выводы, Наука, M., 1972.

[29] S.-I. Amari, Differential Geometrical Methods in Statistics, Lecture Notes in Statist. Vol. 28, Springer, New York, 1985.

[30] Е. А. Морозова, Н. Н. Ченцов, "Марковская инвариантная геометрия на многообразиях состояний”, Итоги науки и техники. Сер. Соврем. пробл. матем., ВИНИТИ, М., 1990, 69-102.

[31] D. Petz, Linear Algebra Appl., 244 (1996), 81-96.

[32] H. Hasegawa, D. Petz, "Non-commutative extension of information geometry", Quantum Communication, Computing and Measurement, ed. H. Hirota, A.S. Holevo, C. M. Caves, Plenum, New York, 1997, 109-118.

[33] D. Petz, G. Toth, Lett. Math. Phys., 27:3 (1993), 205-216.

[34] Ш. Л. Луо, ТМФ, 143:2 (2005), 231-240.

[35] A.D. Sukhanov, Yu. G. Rudoy, "The geometrical aspects of the equilibrium statistical thermodynamics", Proc. of the 5-th Int. Conf. "Bolyai-Gauss-Lobachevsky" (BGL-5) (Minsk, 10-13 Oct. 2006), eds. Yu. Kurochkin, V. Red'kov, Minsk, 2006, 63-75; http://dragon.bas-net.by/bgl5/proc.htm. 